\title{
Nuclear heat shock protein 110 expression is associated with poor prognosis and chemotherapy resistance in gastric cancer
}

\author{
Akiharu Kimura ${ }^{1}$, Kyoichi Ogata ${ }^{1}$, Bolag Altan ${ }^{1}$, Takehiko Yokobori ${ }^{1}$, Munenori Ide ${ }^{2}$, \\ Erito Mochiki ${ }^{3}$, Yoshitaka Toyomasu ${ }^{1}$, Norimichi Kogure ${ }^{1}$, Toru Yanoma ${ }^{1}$, Masaki \\ Suzuki ${ }^{1}$, Tuya Bai ${ }^{1}$, Tetsunari Oyama², Hiroyuki Kuwano ${ }^{1}$ \\ ${ }^{1}$ Department of General Surgical Science, Gunma University Graduate School of Medicine, Maebashi, Gunma, Japan \\ ${ }^{2}$ Department of Diagnostic Pathology, Gunma University Graduate School of Medicine, Maebashi, Gunma, Japan \\ ${ }^{3}$ Department of Digestive Tract and General Surgery, Saitama Medical Center, Saitama Medical University, Kawagoe, Saitama, \\ Japan
}

Correspondence to: Hiroyuki Kuwano, e-mail: hkuwano@gunma-u.ac.jp

Keywords: cancer progression, drug resistance, gastric cancer, heat shock protein, heat shock protein 110

Received: September 25, 2015

Accepted: January 23, 2016

Published: March 01, 2016

\section{ABSTRACT}

Heat shock protein (HSP) expression is induced by the exposure to stress, such as fever, oxidative stress, chemical exposure, and irradiation. In cancer, HSP promotes the survival of malignant cells by inhibiting the induction of apoptosis. In colorectal cancer, a loss-of-function mutation of HSP110 (HSP110 $\triangle E$ 9) has been identified. HSP110 $\triangle E 9$ inhibits the nuclear translocation of wild-type HSP110, which is important for its chaperone activity and anti-apoptotic effects. The patients carrying HSP110 $\triangle$ E9 mutation exhibit high sensitivity to anticancer agents, such as oxaliplatin and 5-fluorouracil.There is still insufficient information about HSP110 localization, the clinicopathological significance of HSP110 expression, and its association with chemotherapy resistance in gastric cancer. Here, we found that high nuclear expression of HSP110 in gastric cancer tissues is associated with cancer progression, poor prognosis, and recurrence after adjuvant chemotherapy. In vitro results showed that HSP110 suppression increases the sensitivity to 5-fluorouracil and cisplatin of human gastric cancer cell lines. Our results suggest that nuclear HSP110 may be a new drug sensitivity marker for gastric cancer and a potential molecular therapeutic target for the treatment of gastric cancer patients with acquired anticancer drug resistance.

\section{INTRODUCTION}

Gastric cancer is one of the most common cancers worldwide and it is particularly prevalent in Asia [1]. Patients with early-stage gastric cancer have a good prognosis following endoscopic or surgical treatment [2], but advanced or recurrent gastric cancer patients have high mortality rates, due to chemotherapy resistance [3]. Therefore, the investigations of the mechanisms of chemotherapy resistance are necessary, in order to improve patient outcomes.

Heat shock proteins (HSPs) are molecular chaperones that facilitate the proper folding and function of proteins. The expression of HSPs is induced by the exposure to stress, such as fever, oxidative stress, chemical exposure, and irradiation $[4,5]$. HSPs provide protection against protein aggregation, facilitate folding of nascent polypeptides, participate in the refolding of proteins that have been damaged, and sequester damaged proteins and target them for degradation [6, 7]. Mammalian HSPs are classified into several protein families based on their molecular weight, namely HSP25/HSP27, HSP40, HSP60, HSP70, HSP90, and HSP110 (also called HSP105) families $[8,9]$. HSP70 family proteins are expressed in the cytoplasm and nucleus of mammalian cells [10]. HSP105 $\alpha$ and HSP $105 \beta$, the alternatively spliced products of HSP 110 family, are expressed in the cytoplasm (HSP105 $\alpha$ ) and in nucleus (HSP105 $\beta$ ) [11]. Previously, it was reported that nuclear HSPs behave as molecular chaperones in cells [10].

HSPs were shown to be overexpressed in a wide range of human carcinomas, including both solid tumors and hematological malignancies [7, 12, 13]. In cancer, HSP promotes the survival of malignant cells by 
protecting several oncoproteins from degradation and inhibiting the induction of apoptosis. This suggests that HSP roles are beneficial for cancerous cells and therefore deleterious for cancer patients [14]. High levels of HSPs may correlate with poor prognosis in several types of cancer. For example, high levels of HSP27 were shown to correlate with poor prognosis in ovarian cancer [15], and HSP60 overexpression was correlated with tumor progression and poor prognosis in colon cancer [16] and prostate carcinoma [17]. The elevated expression of HSP70 is associated with poor prognosis in breast [18] and endometrial [19] cancers, while high HSP90 expression is associated with poor prognosis in invasive ductal breast carcinoma [20] but with good prognosis in endometrial cancer [19]. Furthermore, it was reported that various HSPs, including HSP70 and HSP90, are associated with increased chemosensitivity and may represent potential therapeutic targets in refractory malignancies [21-23].

Antitumor response generated by autologous tumorderived HSP/GRPs (e.g., Hsp70, Hsp90, Grp94/gp96, and calreticulin) has been well documented [9, 24, 25]. Studies over the last decade showed that certain tumor-derived HSPs can serve as effective cancer vaccines, and this has been attributed to an HSP-carried peptide antigenic 'fingerprint' of the tumor [24, 26-29].

Dorard et al. identified a loss-of-function mutation of HSP110 (HSP110 $\mathrm{EE9}$ ) in colorectal cancers with microsatellite instability [30]. HSP110 $\Delta$ E9 lacks a substratebinding domain, and it is unable to play a role of a molecular chaperone for other HSPs (HSP70 or HSP27). Mutant HSP110 $\Delta$ E9 protein associates with wild-type HSP110, blocking its translocation into the nucleus and its chaperone functions. Therefore, HSP110 $\Delta$ E9 overexpression enhances the sensitivity of tumors to anticancer agents, such as oxaliplatin and 5-fluorouracil (5-FU) [30].
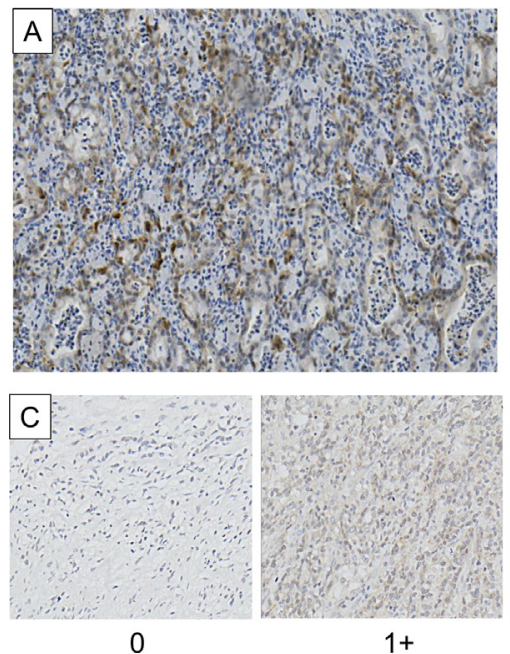

0
However, HSP110 localization, its clinicopathological significance, and its association with chemotherapy resistance in gastric cancer have not been completely elucidated. The objectives of this study were to clarify the significance of HSP110 expression in gastric cancer patients and to assess the effects of HSP110 suppression on chemosensitivity.

\section{RESULTS}

\section{Clinical significance of nuclear HSP110 expression in gastric cancer patients}

Nuclear HSP110 expression was immunohistochemically evaluated using a tissue microarray that included 210 gastric cancer samples. HSP110 expression in cancer tissues was higher compared with non-cancerous tissues. In the cancer tissues, nucleus and cytoplasm were positive for HSP110 immunostaining (Figure 1A and 1B). In non-cancerous tissues, no or weak staining was observed for HSP110. Nuclear HSP110 expression scores in 210 gastric cancer samples were as follows: 0,17 (8.1\%) samples; $1+, 72$ (34.3\%) samples; $2+, 80$ (38.1\%) samples; and 3+, 41(19.5\%) samples. Eighty-nine $(42.4 \%)$ samples were included in the low expression group, and $121(57.6 \%)$ samples were included in the high expression group. The relationship of nuclear HSP110 expression and clinicopathological factors from 210 gastric cancer patients is presented in Table 1. High nuclear expression of HSP110 was significantly associated with venous invasion $(P=0.0464)$. The overall survival rate in the high expression group was significantly lower compared with the low expression group ( $P=0.0169$; Figure 2$)$. Multivariate regression analysis revealed that high expression of nuclear
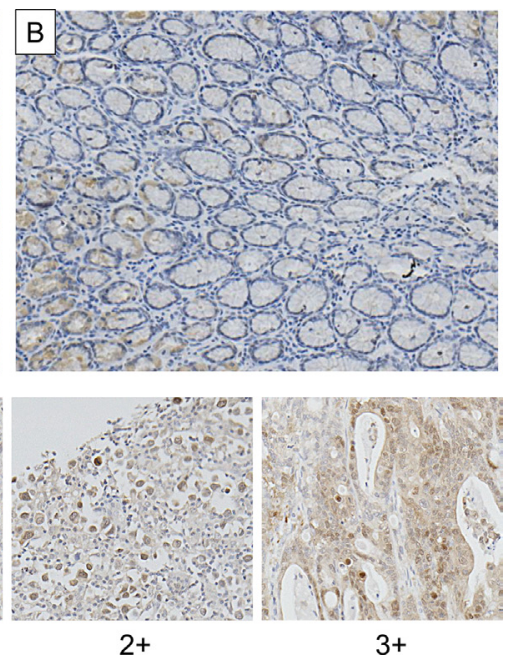

Figure 1: Immunohistochemical staining of HSP110 in primary gastric cancer samples. (A) Cancerous tissue; (B) Noncancerous tissue (original magnification, $\times 200$ ). (C) Tissue microarray samples (original magnification, $200 \times$ ). The intensity of nuclear HSP110 staining was scored as follows: 0 , no staining; $1+$, weak staining; $2+$, moderate staining; $3+$, strong staining. 
Table 1: The relationship between clinicopathological characteristics of gastric cancer patients and the nuclear HSP110 expression levels

\begin{tabular}{|c|c|c|c|}
\hline \multirow{2}{*}{ Factors } & \multicolumn{3}{|c|}{ HSP110 expression in gastric cancer $(n=210)$} \\
\hline & Low $(n=89)$ & High $(n=121)$ & $P$ value \\
\hline Age (mean \pm standard error) & $63.1 \pm 1.2$ & $65.7 \pm 1.0$ & 0.1007 \\
\hline \multicolumn{4}{|l|}{ Gender, $n(\%)$} \\
\hline Male & $66(44.9 \%)$ & $81(55.1 \%)$ & 0.2574 \\
\hline Female & $23(36.5 \%)$ & $40(63.5 \%)$ & \\
\hline \multicolumn{4}{|l|}{ Histology, $n(\%)$} \\
\hline Well, Moderate & $33(41.8 \%)$ & $46(58.2 \%)$ & 0.8897 \\
\hline Poor, Signet & $56(42.7 \%)$ & $75(57.3 \%)$ & \\
\hline \multicolumn{4}{|l|}{ Depth, $n(\%)$} \\
\hline $\mathrm{sm}, \mathrm{mp}, \mathrm{ss}$ & $48(40.7 \%)$ & $70(59.3 \%)$ & 0.8266 \\
\hline se, si & $41(44.6 \%)$ & $51(55.4 \%)$ & \\
\hline \multicolumn{4}{|l|}{ Lymph node metastasis, $n(\%)$} \\
\hline Absent & $30(44.8 \%)$ & $37(55.2 \%)$ & 0.6311 \\
\hline Present & $59(41.3 \%)$ & $84(58.7 \%)$ & \\
\hline \multicolumn{4}{|l|}{ Lymphatic invasion, $n(\%)$} \\
\hline Absent & $9(47.4 \%)$ & $10(52.6 \%)$ & 0.6459 \\
\hline Present & $80(41.9 \%)$ & $111(58.1 \%)$ & \\
\hline \multicolumn{4}{|l|}{ Venous invasion, $n(\%)$} \\
\hline Absent & $71(46.7 \%)$ & $81(53.3 \%)$ & $0.0464^{*}$ \\
\hline Present & $18(31.6 \%)$ & $39(68.4 \%)$ & \\
\hline \multicolumn{4}{|l|}{ Stage, $n(\%)$} \\
\hline I & $12(36.4 \%)$ & $21(63.6 \%)$ & 0.5754 \\
\hline II & $33(47.8 \%)$ & $36(52.2 \%)$ & \\
\hline III & $35(42.7 \%)$ & $47(57.3 \%)$ & \\
\hline IV & $9(34.6 \%)$ & $17(65.4 \%)$ & \\
\hline
\end{tabular}

$* P<0.05$.

Well: well differentiated, Moderate: moderately differentiated, Poor: poorly differentiated, Signet: signet ring cells, sm: submucosa, mp: muscularis propria, ss: subserosa, se: serosa exposed, si: serosa infiltrating.

HSP110 is an independent prognostic factor for gastric cancer outcome $(P=0.0068)$, as are the tumor depth $(P<0.001)$ and venous invasion $(P=0.0276$; Table 2). Additionally, we assessed cytoplasmic HSP110 expression in 210 gastric cancer tissue samples. No significant difference in prognosis was observed between the cytoplasmic HSP110 high expression group and low expression group $(P=0.6884$, Supplementary Figure S1). Furthermore, additional analysis was performed in order to evaluate the significance of total HSP110 expression in gastric cancer tissues. The high cytoplasmic and nuclear HSP110 expression groups were defined as total HSP110 high expression group $(n=74)$. The low cytoplasmic and nuclear HSP110 expression groups were defined as total HSP110 low expression group $(n=73)$. There was no significant prognostic difference between the total HSP110 high expression group and low expression group $(P=0.2021$, Supplementary Figure S2). The relationship between total HSP110 expression and clinicopathological factors is shown in Supplementary Table S1. No significant relationships were found between total HSP110 expression and clinicopathological factors, with the only exception being patients' ages. 


\section{Prognostic significance of nuclear HSP110 expression in gastric cancer patients who received adjuvant chemotherapy}

Forty-eight of the 210 gastric cancer patients received 5-FU-based adjuvant chemotherapy. We evaluated the correlation between nuclear HSP110 expression and prognosis in these patients (Figure 3A and 3B). Among patients who received adjuvant chemotherapy, the overall survival rate in the high expression group was significantly lower compared with the low expression group $(P=0.0364)$. There was no significant difference in disease-free survival between the two groups, but the disease-free survival rate in the high expression group tended to be lower compared with the low expression group $(P=0.0743)$.

\section{HSP110 expression in gastric cancer cell lines}

HSP110 expression was detected using western blot in all human gastric cancer cell lines (MKN7, MKN45, MKN74, AZ521) (Figure 4A). MKN7 and MKN45 were further used for the in vitro analyses of the effects of HSP110 suppression in gastric cancer cell lines. HSP110 expression was suppressed in MKN7 and MKN45 cells treated with HSP110 siRNA (Figure 4B and 4C).

\section{The effects of HSP110 suppression on the chemosensitivity of gastric cancer cell lines}

We evaluated the correlation between HSP110 suppression and the chemosensitivity of gastric cancer cell lines. Following HSP110 knockdown, MKN7 and MKN45 cells were treated with 5-FU or cisplatin. The sensitivity to 5-FU and cisplatin of HSP110 siRNA-treated cells was significantly higher compared with the parent and control cells $(P<0.05$; Figure 4D).

\section{DISCUSSION}

Here, we determined that high nuclear expression of HSP110 in gastric cancer tissues is associated with cancer progression and poor prognosis. Among patients who received adjuvant chemotherapy, those included in the high HSP110 expression group showed significantly shorter overall survival compared with the low expression group. In vitro study showed that HSP110 suppression increases the sensitivity to 5-FU and cisplatin in human gastric cancer cell lines.

Immunohistochemical analysis demonstrated that the high expression of nuclear HSP110 is associated with poor overall survival. Based on the multivariate analysis of the factors affecting overall survival, the high expression of nuclear HSP110 was shown to be an independent prognostic factor. Previously, it was reported that the high expression of HSP110 is associated with poor prognosis in lung adenocarcinoma [31] and colorectal cancer [32], and our results are consistent with these reports. The chaperoning properties of HSP110 are integral to the ability of these molecules to modulate immune functions and for the development of large chaperone complex vaccines for cancer immunotherapy [9]. Nakajima et al. reported that high cytoplasmic HSP110 expression induces CD4+ T lymphocyte infiltration, which was shown to be associated with good prognosis in esophageal cancer [33]. We assessed total and cytoplasmic HSP110 expression

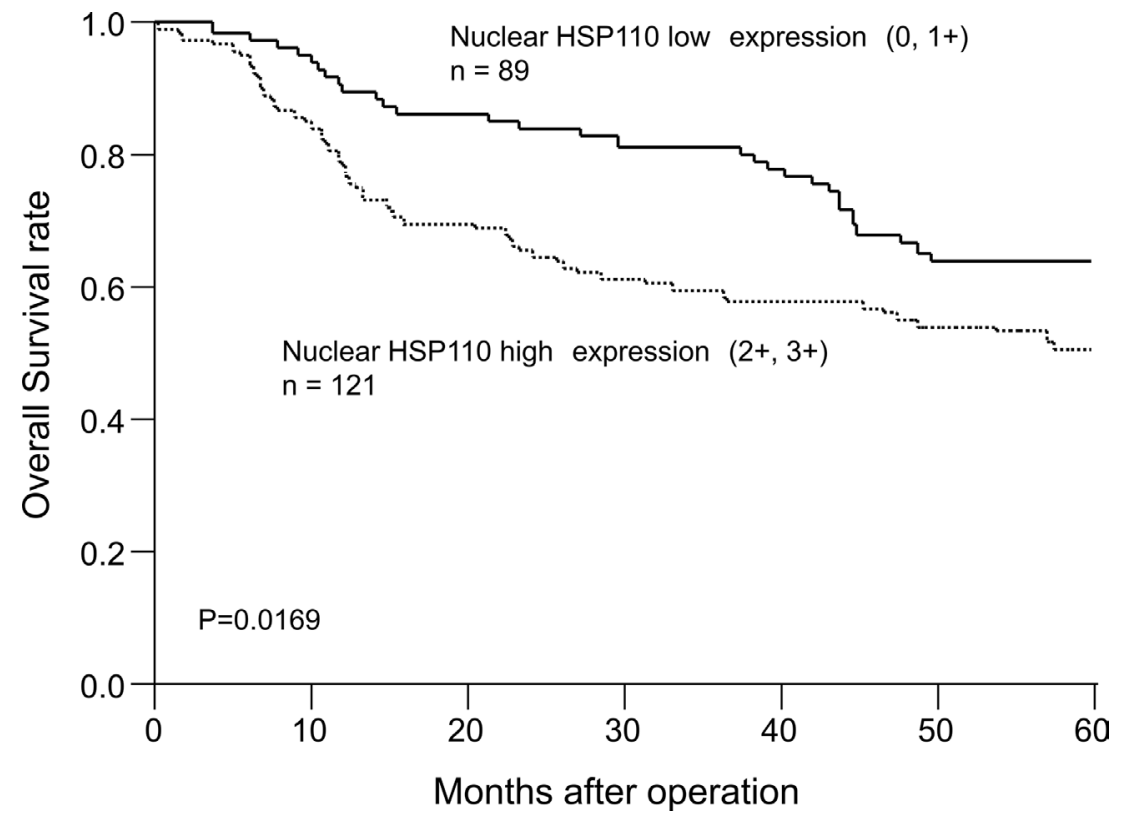

Figure 2: Overall survival of gastric cancer patients according to the nuclear HSP110 expression. The overall survival in the nuclear HSP110 high expression group was significantly shorter compared with the low expression group $(P=0.0169)$. 
Table 2: Univariate and multivariate analyses of clinicopathological factors affecting overall survival rates after surgery

\begin{tabular}{|l|c|c|c|c|c|c|}
\hline \multirow{2}{*}{\multicolumn{1}{c}{ Clinicopathological variables }} & \multicolumn{3}{c}{ Univariate analysis } & \multicolumn{3}{c}{ Multivariate analysis } \\
\cline { 2 - 7 } \multicolumn{1}{c}{} & RR & $\mathbf{9 5 \%}$ CI & P value & RR & 95\% CI & \multicolumn{1}{c}{$\boldsymbol{P}$ value } \\
\hline Age $(<65$ years/ $\geq 65$ years) & 1.11 & $0.90-1.37$ & 0.3209 & - & - & - \\
\hline Gender (male/female) & 0.9 & $0.71-1.13$ & 0.3873 & - & - & - \\
\hline Histology (differentiated/undifferentiated) & 1.11 & $0.89-1.38$ & 0.3546 & - & - & - \\
\hline Depth (sm, mp, ss/se, si) & 1.83 & $1.48-2.29$ & $0.0000^{*}$ & 1.74 & $1.39-2.19$ & $<0.001 *$ \\
\hline Lymph node metastasis (absent/present) & 1.59 & $1.24-2.11$ & $0.0002 *$ & 1.31 & $0.99-1.78$ & 0.0579 \\
\hline Lymphatic invasion (absent/present) & 1.88 & $1.15-3.79$ & $0.0081 *$ & 1.12 & $0.64-2.33$ & 0.7230 \\
\hline Venous invasion (absent/present) & 1.47 & $1.19-1.83$ & $0.006^{*}$ & 1.29 & $1.03-1.60$ & $0.0276^{*}$ \\
\hline HSP110 expression (low/high) & 1.3 & $1.05-1.63$ & $0.0155^{*}$ & 1.35 & $1.09-1.70$ & $0.0068^{*}$ \\
\hline
\end{tabular}

$* P<0.05$.

RR: Relative risk, CI: Confidence interval, sm: submucosa, mp: muscularis propria, ss: subserosa, se: serosa exposed, si: serosa infiltrating.

in gastric cancer samples, but no significant prognostic differences were observed in the total and cytoplasmic HSP110 expression between the high and low groups (Supplementary Figures S1, S2). It was previously reported that wild-type nuclear, rather than cytoplasmic, HSP110 prevents the induction of apoptosis in colorectal cancer cells [30]. Therefore, we suggest that nuclear HSP110 expression levels may be a useful prognostic and drug sensitivity marker for gastric cancer.

A

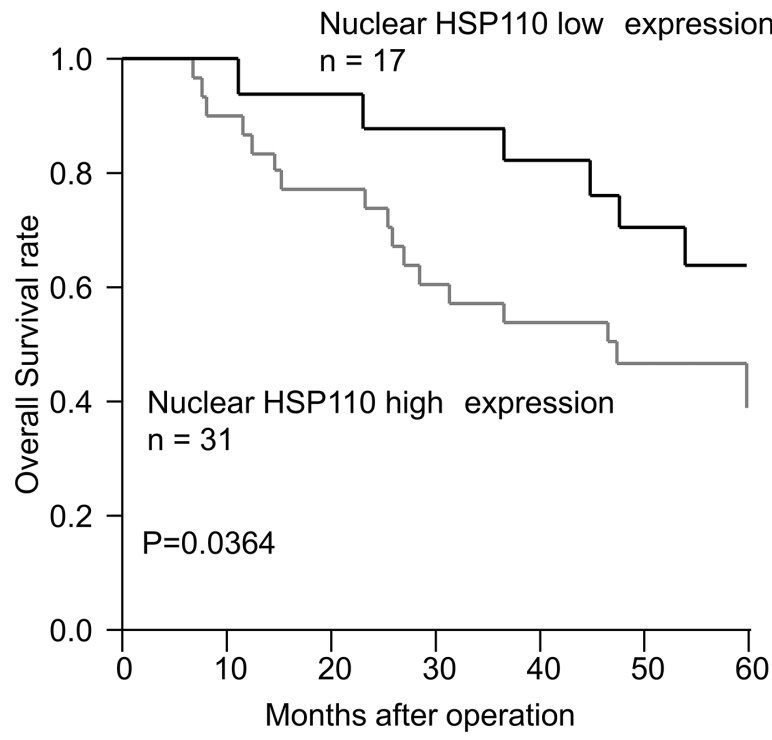

In this study, the high expression of nuclear HSP110 was shown to be associated with venous invasion (Table 1). HSPs promote cancer progression in several cancer types, and Gong et al. [34] reported that the invasion potential of hepatocarcinoma cells is increased by HMGB1-induced tumor NF- $\kappa$ B signaling, through the activation of HSP70. Sims et al. [35] reported that extracellular HSP70 and HSP90 $\alpha$ contribute to the matrix metalloproteinase- 2 activation and breast cancer cell migration and invasion.

B

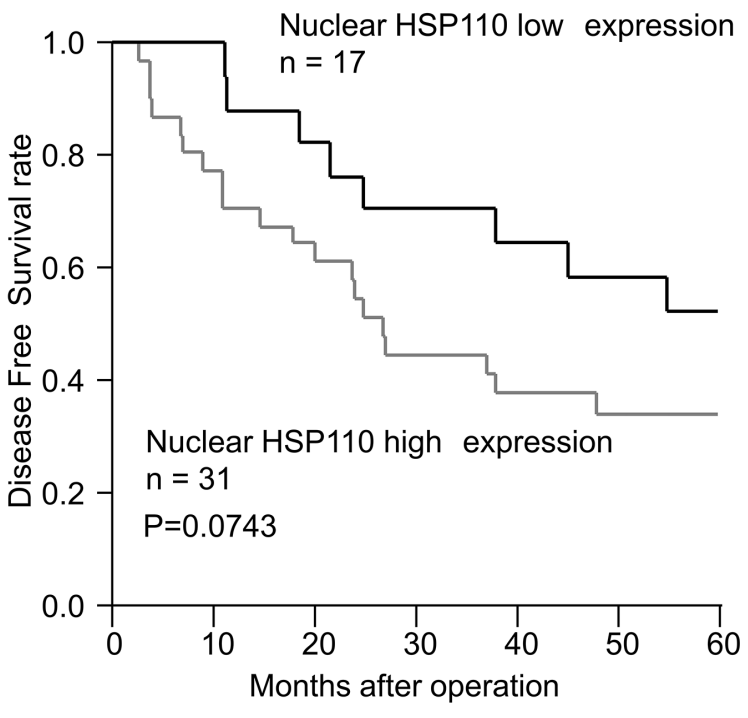

Figure 3: The survival curves of gastric cancer patients who received adjuvant chemotherapy according to nuclear HSP110 expression. (A) Overall survival. (B) Disease-free survival. Among the patients who received adjuvant chemotherapy, the overall survival rate in the nuclear HSP110 high expression group was significantly lower compared with the low expression group $(P=0.0364)$. No significant difference in disease-free survival was observed between these groups; however, the disease-free survival rate in the high expression group tended to be lower compared with the low expression group $(P=0.0743)$. 
It has also been reported that HSP110 is co-expressed with HSP70 and HSP90 during stress, and that it promotes HSP90 activity and may function as a nucleotide exchange factor for cytosolic HSP70 [36]. We elucidated whether HSP110 expression can facilitate cancer invasion through the activation of HSP70 and HSP90.

Hosaka et al. [37] reported that HSP110 suppression induces apoptosis in cancer cell lines but not in fibroblasts. Dorard et al. [30] identified a loss-of-function mutation of HSP110 (HSP110 $\Delta \mathrm{E} 9$ ) in colorectal cancer with microsatellite instability. HSP110 $\mathrm{E} 9$ overexpression enhanced cancer cell sensitivity to anticancer agents. Here, low nuclear HSP110 expression group had better prognosis compared with the high expression group, and HSP110 suppression was shown to increase cell sensitivity to 5-FU and cisplatin in human gastric cancer cell lines, which is consistent with the previous reports. Novel treatment strategies, combining an HSP110 inhibitor and an anticancer agent, may be effective for the treatment of gastric cancer patients with acquired anticancer drug resistance.

Previously, HSP110 was identified as a cancer antigen in various human carcinomas $[31,32]$. We report here that the high expression of the nuclear HSP110 was observed in gastric cancer patients. Wang et al. [29] developed a vaccine composed of a recombinant protein coupled with large heat shock protein. Our results suggest that chemosensitivity may decrease due to heat stressinduced HSP110 expression. Therefore, various vaccines, which may utilize HSPs (i.e., covalent coupling, isolation of HSPs with antigens attached, recombinant vaccines made by heat-denaturation of full-length antigens and HSP110) may be useful in anticancer treatments.

HSP110-specific siRNAs were used to suppress the expression of HSP110 in gastric cancer cell lines, which presents a limitation of this study. Therefore, total HSP110 expression was suppressed, and not only the specific nuclear HSP110 expression. Total HP110 expression was strongly suppressed in MKN7 and MKN45 by the HSP110-specific siRNA, but this is probably the consequence of the suppression of both nuclear and cytoplasmic HSP110 expression.

In conclusion, the high expression of nuclear HSP110 was shown to be associated with cancer progression, poor prognosis, and recurrence after adjuvant
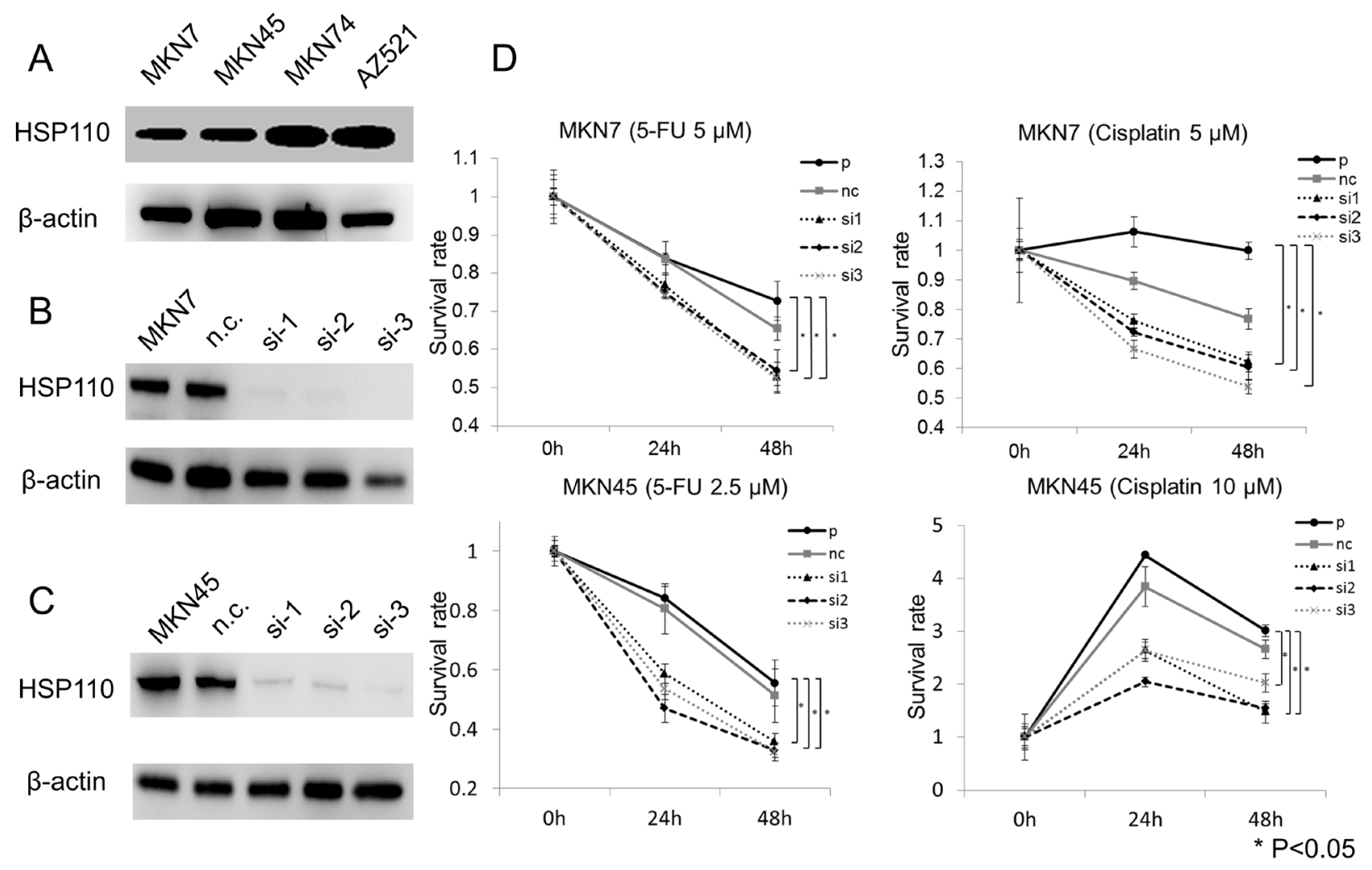

n.c.: negative control (scrambled siRNA) si: siRNA

Figure 4: Functional analysis of human gastric cancer cell lines treated with HSP110 siRNA. (A) The expression of HSP110 in human gastric cancer cell lines was assessed by western blot. $\beta$-actin was used as the loading control. (B) HSP110 expression was suppressed using HSP110 siRNA (MKN7); (C) HSP110 suppression using HSP110 siRNA (MKN45). (D) The effects of HSP110 suppression on chemosensitivity of MKN7 and MKN45 cells. Both MKN7 and MKN45 cells showed a significantly increased sensitivity to 5-fluorouracil in HSP110 siRNA-treated groups, compared with the parent and control cells $(P<0.05)$. n.c.: negative control (scrambled siRNA), si: siRNA. 
chemotherapy in gastric cancer patients. Furthermore, HSP110 suppression increased the sensitivity to 5-FU and cisplatin in the human gastric cancer cell lines. Our results suggest that nuclear HSP110 expression in gastric cancer may be a new prognostic and drug sensitivity marker, and HSP110 may serve as a new molecular therapeutic target for the treatment of refractory gastric cancer.

\section{METHODS}

\section{Patients and samples}

Primary gastric cancer tissues were obtained from gastric cancer patients ( $n=210 ; 147$ men and 63 women) who underwent radical gastrectomy at the Department of General Surgical Science, Gunma University Hospital, Japan, between January 1999 and May 2006. The stage of gastric cancer was described according to the classification of gastric carcinoma of the Japanese Gastric Cancer Association's 3rd English edition [38]. Forty-eight patients received 5-FU-based adjuvant chemotherapy between January 2003 and May 2006. The correlation between HSP110 expression and clinicopathological factors and prognosis was evaluated in these patients. Written informed consents were obtained from all patients according to institutional guidelines.

\section{Tissue microarray analysis and immunohistochemical staining}

Tumor samples were fixed in formalin, embedded in paraffin, and stored in the archives of the Clinical Department of Pathology, Gunma University Hospital, Japan. For 210 gastric cancer patients, one paraffin block containing representative non-necrotic tumor areas was selected, and gastric cancer tissue cores $(2.0 \mathrm{~mm}$ diameter per tumor) were sampled from the representative areas and transferred into the paraffin block using a tissue arraying instrument (Beecher Instruments, Silver Spring, MD, USA). Cores were arranged into quad tissue array blocks, with each containing 50-55 tumor cores. Tissue microarray blocks were cut into $3.5-\mu \mathrm{m}$ thick sections, and were used for the subsequent immunohistochemical staining. Additionally, 4- $\mu \mathrm{m}$ sections were cut from the paraffin blocks of 10 gastric cancer samples, selected among 210 gastric cancer patients for validation.

All sections were incubated at $60^{\circ} \mathrm{C}$ for $60 \mathrm{~min}$ and deparaffinized in xylene. Afterward, these sections were rehydrated and incubated with fresh $0.3 \%$ hydrogen peroxide in $100 \%$ methanol for $30 \mathrm{~min}$ at room temperature, in order to block endogenous peroxidase activity. Following the rehydration through a graded series of ethanol treatments, the sections were heated in boiling water and soaked in Immunosaver (Nishin EM, Tokyo, Japan) at $98^{\circ} \mathrm{C}$ for $90 \mathrm{~min}$. Non-specific binding sites were blocked by incubating the sections with Protein Block Serum-Free (DAKO, Carpinteria, CA, USA) for 30 min. A rabbit monoclonal anti-HSP110 antibody (GeneTex, CA, USA) was applied at 1:100 dilution, for $24 \mathrm{~h}$ at $4^{\circ} \mathrm{C}$. The primary antibody was visualized using the Histofine Simple Stain MAX-PO (MULTI) (Nichirei, Tokyo, Japan) according to the instruction manual. A chromogen, 3,3-diaminobenzidine tetrahydrochloride, was applied as a $0.02 \%$ solution containing $0.005 \%$ hydrogen peroxide in $50 \mathrm{mM}$ ammonium acetate-citrate acid buffer ( $\mathrm{pH}$ 6.0). The sections were lightly counterstained with Mayer's hematoxylin and mounted. The evaluation of immunohistochemical staining was performed by two independent researchers who were blinded to the patients' data. We focused on nuclear HSP110 expression, and the intensity of nuclear HSP110 staining was scored as follows: 0 , no staining; $1+$, weak staining; $2+$, moderate staining; $3+$, strong staining. Gastric cancer patients were assigned to the nuclear HSP110 low expression group $(0,1+)$ or high expression group $(2+, 3+)$, according to staining score (Figure 1C). Additionally, the tissues adjacent to the cancerous tissues in the tissue microarray samples were considered non-cancerous tissue. We evaluated the expression of HSP110 in the non-cancerous tissue of these 10 samples, for validation. The non-cancerous tissue was defined as the normal gastric mucosa tissue or stromal cells.

\section{Cell culture}

The human gastric cancer cell lines MKN7, MKN45, MKN74, and AZ521 were used in this study. These cell lines were obtained from RIKEN BRC through the National Bio-Resource Project of MEXT, Tokyo, Japan. The cells were cultured in RPMI 1640 medium (Wako, Osaka, Japan) supplemented with 10\% FBS and $1 \%$ penicillin-streptomycin (Invitrogen, Carlsbad, CA, USA).

\section{siRNA transfection}

HSP110-specific siRNA was purchased from Bonac Corporation (Fukuoka, Japan). MKN7 and MKN45 cells were plated at a density of $1.0 \times 10^{6}$ cells per well in $100 \mu \mathrm{l}$ of Opti-MEM I Reduced Serum Medium (Invitrogen, Carlsbad, CA, USA). Twenty nM of HSP110-specific siRNA 1, 2, 3 and scrambled siRNA (negative control) were added to the cells, and cells were transfected with siRNAs using an electroporator (CUY-21 EDIT II; BEX, Tokyo, Japan), according to the manufacturer's instructions. Poring pulses were applied at $125 \mathrm{~V}$ (pulse length, $10.0 \mathrm{~ms}$; 1 pulse; interval, $40.0 \mathrm{~ms}$ ), and transfer pulses were applied at $10 \mathrm{~V}$ (pulse length, $50.0 \mathrm{~ms}$; 5 pulses; interval, $50.0 \mathrm{~ms}$ ). After $72 \mathrm{~h}$ of incubation, further experiments were performed. 


\section{Protein extraction and western blot analysis}

Western blotting was performed to confirm the expression of HSP110 and $\beta$-actin in gastric cancer cell lines. Transfected cells were incubated for $72 \mathrm{~h}$, and total proteins were extracted from MKN7, MKN45, MKN74, and AZ521 cells using PRO-PREP Protein Extraction Solution Kit (iNtRON Biotechnology, Sungnam, KyungkiDo, Korea). The proteins were separated on $4-12 \%$ Bis-Tris Mini Gels (Life Technologies Corporation, Carlsbad, CA, USA), and transferred to membranes using an iBlot Dry Blotting System (Life Technologies Corporation, Carlsbad, CA, USA). The membranes were incubated overnight at $4{ }^{\circ} \mathrm{C}$ with rabbit monoclonal antiHSP110 antibody (1:1000; GeneTex, CA, USA) and anti- $\beta$-actin antibody (1:1000; Sigma-Aldrich, St Louis, MO, USA). Following this, the membranes were incubated with horseradish peroxidase-conjugated anti-rabbit secondary antibodies, and the target proteins were detected with the ECL Prime Western Blotting Detection System (GE Healthcare, Tokyo, Japan) using Image Quant LAS4000 (GE Healthcare Life Sciences, UK).

\section{Chemosensitivity assay}

Water-soluble tetrazolium-8 (Cell Counting Kit-8; Dojindo Laboratories, Japan) was used in order to evaluate the sensitivity to cisplatin and 5-FU. After $72 \mathrm{~h}$ of incubation following the transfection, MKN7 and MKN45 cells were seeded $\left(1 \times 10^{4}\right.$ cells/well $)$ into 96 -well plates in $100 \mu$ of RPMI 1640 medium containing 20\% FBS before drug exposure. After $24 \mathrm{~h}$ of pre-incubation, $10 \mu \mathrm{l}$ of Cell Counting Kit- 8 reagent were added, and the cells were additionally incubated for $2 \mathrm{~h}$ at $37^{\circ} \mathrm{C}$. The absorbance of each well was detected at $450 \mathrm{~nm}$ using an xMark Microplate Absorbance Spectrophotometer (Bio Rad, Hercules, CA, USA). Afterward, the cells were treated with various concentrations of cisplatin and 5-FU for $48 \mathrm{~h}$. Viability was determined using colorimetry by measuring absorbance every $24 \mathrm{~h}$.

\section{Statistical analysis}

Data for continuous variables were expressed as mean \pm standard error of the mean. Significance was determined using Student's $t$-test and analysis of variance. The statistical analysis of the immunohistochemical staining results was performed using the chi-squared test. Survival curves were generated according to the Kaplan-Meier method and analyzed using the log-rank test. Prognostic factors were examined by univariate and multivariate analyses using a Cox proportional hazards model. Results were considered statistically significant when $P$ value was $<0.05$. All statistical analyses were performed using JMP software, version 12 (SAS Institute Inc., Cary, NC, USA).

\section{ACKNOWLEDGMENTS AND FUNDING}

The authors thank Ms. Yukie Saito, Ms. Tomoko Yano, Ms. Tomoko Ubukata, Ms. Yuka Matsui, Ms. Ayaka Ishida, and Ayaka Ishikubo for their excellent assistance.

\section{CONFLICTS OF INTEREST}

None.

\section{REFERENCES}

1. Ferlay J, Shin HR, Bray F, Forman D, Mathers C, Parkin DM. Estimates of worldwide burden of cancer in 2008: GLOBOCAN 2008. Int J Cancer. 2010; 127:2893-2917.

2. Uedo $N$, Iishi $H$, Tatsuta $M$, Ishihara $R$, Higashino $K$, Takeuchi Y, Imanaka K, Yamada T, Yamamoto S, Tsukuma H, Ishiguro S. Longterm outcomes after endoscopic mucosal resection for early gastric cancer. Gastric Cancer. 2006; 9:88-92.

3. Cervantes A, Roselló S, Roda D, Rodríguez-Braun E. The treatment of advanced gastric cancer: current strategies and future perspectives. Ann Oncol. 2008; 19 Suppl 5: v103-107.

4. Lindquist S, Craig EA. The heat-shock proteins. Annu Rev Genet. 1988; 22:631-677.

5. Young JC, Agashe VR, Siegers K, Hartl FU. Pathways of chaperone-mediated protein folding in the cytosol. Nat Rev Mol Cell Biol. 2004; 5:781-791.

6. Hartl FU. Molecular chaperones in cellular protein folding. Nature. 1996; 381:571-579.

7. Soo ET, Yip GW, Lwin ZM, Kumar SD, Bay BH. Heat shock proteins as novel therapeutic targets in cancer. in vivo. 2008; 22:311-315.

8. Subjeck JR, Shyy TT. Stress protein systems of mammalian cells. Am J Physiol. 1986; 250:C1-17.

9. Wang XY, Subjeck JR. High molecular weight stress proteins: Identification, cloning and utilisation in cancer immunotherapy. Int J Hyperthermia. 2013; 29:364-375.

10. Welch WJ, Feramisco JR. Nuclear and nucleolar localization of the 72,000-dalton heat shock protein in heat-shocked mammalian cells. J Biol Chem. 1984; 259:4501-4513.

11. Saito Y, Yamagishi N, Hatayama T. Different localization of Hsp105 family proteins in mammalian cells. Exp Cell Res. 2007; 313:3707-3717.

12. Whitesell L, Lindquist SL. HSP90 and the chaperoning of cancer. Nat Rev Cancer. 2005; 5:761-772.

13. Chant ID, Rose PE, Morris AG. Analysis of heat-shock protein expression in myeloid leukaemia cells by flow cytometry. Br J Haematol. 1995; 90:163-168.

14. Rappa F, Farina F, Zummo G, David S, Campanella C, Carini F, Tomasello G, Damiani P, Cappello F, DE Macario EC, Macario AJ. HSP-molecular chaperones in cancer biogenesis 
and tumor therapy: an overview. Anticancer Res. 2012; 32:5139-5150.

15. Langdon SP, Rabiasz GJ, Hirst GL, King RJ, Hawkins RA, Smyth JF, Miller WR. Expression of the heat shock protein HSP27 in human ovarian cancer. Clin Cancer Res. 1995; $1: 1603-1609$.

16. Cappello F, David S, Rappa F, Bucchieri F, Marasà L, Bartolotta TE, Farina F, Zummo G. The expression of HSP60 and HSP10 in large bowel carcinomas with lymph node metastase. BMC Cancer. 2005; 5:139.

17. Cappello F, Rappa F, David S, Anzalone R, Zummo G. Immunohistochemical evaluation of PCNA, p53, HSP60, HSP10 and MUC-2 presence and expression in prostate carcinogenesis. Anticancer Res. 2003; 23:1325-1331.

18. Lazaris ACh, Chatzigianni EB, Panoussopoulos D, Tzimas GN, Davaris PS, Golematis BCh. Proliferating cell nuclear antigen and heat shock protein 70 immunolocalization in invasive ductal breast cancer not otherwise specified. Breast Cancer Res Treat. 1997; 43:43-51.

19. Nanbu K, Konishi I, Komatsu T, Mandai M, Yamamoto S, Kuroda H, Koshiyama M, Mori T. Expression of heat shock proteins HSP70 and HSP90 in endometrial carcinomas. Correlation with clinicopathology, sex steroid receptor status, and p53 protein expression. Cancer. 1996; 77:330-338.

20. Pick E, Kluger Y, Giltnane JM, Moeder C, Camp RL, Rimm DL, Kluger HM. High HSP90 expression is associated with decreased survival in breast cancer. Cancer Res. 2007; 67:2932-2937.

21. He LF, Guan KP, Yan Z, Ye HY, Xu KX, Ren L, Hou SK. Enhanced sensitivity to mitomycin $\mathrm{C}$ by abating heat shock protein 70 expression in human bladder cancer cell line of BIU-87. Chin Med J (Engl). 2005; 118:1965-1972.

22. Lai CH, Park KS, Lee DH, Alberobello AT, Raffeld M, Pierobon M, Pin E, Petricoin Iii EF, Wang Y, Giaccone G. HSP-90 inhibitor ganetespib is synergistic with doxorubicin in small cell lung cancer. Oncogene. 2014; 33:4867-4876.

23. Fang X, Jiang Y, Feng L, Chen H, Zhen C, Ding M, Wang X. Blockade of PI3K/AKT pathway enhances sensitivity of Raji cells to chemotherapy through down-regulation of HSP70. Cancer Cell Int. 2013; 13:48.

24. Tamura Y, Peng P, Liu K, Daou M, Srivastava PK. Immunotherapy of tumors with autologous tumor-derived heat shock protein preparations. Science. 1997; 278: 117-120.

25. Vanaja DK, Grossmann ME, Celis E, Young CY. Tumor prevention and antitumor immunity with heat shock protein 70 induced by 15-deoxy-delta12, 14-prostaglandin J2 in transgenic adenocarcinoma of mouse prostate cells. Cancer Res. 2000; 60:4714-4718.

26. Graner M, Raymond A, Romney D, He L, Whitesell L, Katsanis E. Immunoprotective activities of multiple chaperone proteins isolated from murine B-cell leukemia/ lymphoma. Clin Cancer Res. 2000; 6:909-915.
27. Wang XY, Kazim L, Repasky EA, Subjeck JR. Characterization of heat shock protein 110 and glucoseregulated protein 170 as cancer vaccines and the effect of fever-range hyperthermia on vaccine activity. J Immunol. $2001 ; 166: 490-497$.

28. Srivastava P. Interaction of heat shock proteins with peptides and antigen presenting cells: chaperoning of the innate and adaptive immune responses. Annu Rev Immunol. 2002; 20:395-425.

29. Wang XY, Yi H, Yu X, Zuo D, Subjeck JR. Enhancing antigen cross-presentation and T-cell priming by complexing protein antigen to recombinant large heat-shock protein. Methods Mol Biol. 2011; 787:277-287.

30. Dorard C, de Thonel A, Collura A, Marisa L, Svrcek M, Lagrange A, Jego G, Wanherdrick K, Joly AL, Buhard O, Gobbo J, Penard-Lacronique V, Zouali H, et al. Expression of a mutant HSP110 sensitizes colorectal cancer cells to chemotherapy and improves disease prognosis. Nat Med. 2011; 17:1283-1289.

31. Oda T, Morii E, Inoue M, Ikeda J, Aozasa K, Okumura M. Prognostic significance of heat shock protein 105 in lung adenocarcinoma. Mol Med Rep. 2009; 2:603-607.

32. Slaby O, Sobkova K, Svoboda M, Garajova I, Fabian P, Hrstka R, Nenutil R, Sachlova M, Kocakova I, Michalek J, Smerdova T, Knoflickova D, Vyzula R. Significant overexpression of Hsp110 gene during colorectal cancer progression. Oncol Rep. 2009; 21:1235-1241.

33. Nakajima M, Kato H, Miyazaki T, Fukuchi M, Masuda N, Fukai Y, Sohda M, Inose T, Sakai M, Sano A, Tanaka N, Ahmad F, Kuwano H. Prognostic significance of heat shock protein 110 expression and T lymphocyte infiltration in esophageal cancer. Hepatogastroenterology. 2011; 58: 1555-1560.

34. Gong W, Wang ZY, Chen GX, Liu YQ, Gu XY, Liu WW. Invasion potential of $\mathrm{H} 22$ hepatocarcinoma cells is increased by HMGB1-induced tumor NF- $\mathrm{BB}$ signaling via initiation of HSP70. Oncol Rep. 2013; 30:1249-1256.

35. Sims JD, McCready J, Jay DG. Extracellular heat shock protein (Hsp)70 and Hsp90 $\alpha$ assist in matrix metalloproteinase-2 activation and breast cancer cell migration and invasion. PLoS One. 2011; 6:e18848.

36. Mandal AK, Gibney PA, Nillegoda NB, Theodoraki MA, Caplan AJ, Morano KA. Hsp110 chaperones control client fate determination in the hsp70-Hsp90 chaperone system. Mol Biol Cell. 2010; 21:1439-1448.

37. Hosaka S, Nakatsura T, Tsukamoto H, Hatayama T, Baba H, Nishimura Y. Synthetic small interfering RNA targeting heat shock protein 105 induces apoptosis of various cancer cells both in vitro and in vivo. Cancer Sci. 2006; 97:623-632.

38. Association JGC. Japanese classification of gastric carcinoma: 3rd English edition. Gastric Cancer. 2011; 14:101-112. 